\title{
Fluorescence-Imaged Microdeformation of the Outer Hair Cell Lateral Wall
}

\author{
John S. Oghalai, ${ }^{1}$ Alpen A. Patel, ${ }^{1}$ Takashi Nakagawa, ${ }^{1,2}$ and William E. Brownell ${ }^{1}$ \\ ${ }^{1}$ Bobby R. Alford Department of Otorhinolaryngology and Communicative Sciences, Baylor College of Medicine, \\ Houston, Texas 77030, and 2Department of Otorhinolaryngology, Faculty of Medicine, Kyushu University, \\ Fukuoka 812, Japan
}

Outer hair cell $(\mathrm{OHC})$ electromotility appears to be central to mammalian hearing and originates within its lateral wall. The $\mathrm{OHC}$ lateral wall is a unique trilaminate structure consisting of the plasma membrane (PM), the cortical lattice (CL), and the subsurface cisternae (SSC). We selectively labeled and imaged the lateral wall components in the isolated guinea pig $\mathrm{OHC}$ under confocal microscopy. The PM was labeled with a voltage-sensitive dye, di-8-ANEPPS; the SSC was labeled with the sphingomyelin precursor, $\mathrm{NBD}_{-} \mathrm{C}_{6}$-ceramide; and F-actin in the $\mathrm{CL}$ was labeled with conjugates of phalloidin. Interactions among the three layers were evaluated with the micropipette aspiration technique. The PM was tethered to the CL and SSC until, at a critical deformation pressure, the PM separated, allowing visualization of the extracisternal space, and ultimately formed a vesicle. After detaching, the stiffness parameter of the PM was $22 \%$ of that of the intact lateral wall. We conclude that the lateral wall PM is more compliant than the CL/SSC complex. The data clarify the structural basis for electromotile force coupling in the $\mathrm{OHC}$ lateral wall.

Key words: cochlea; inner ear; hearing; cytoskeleton; cell stiffness; stiffness parameter; micropipette aspiration; patchclamp technique; confocal microscopy; fluorescence labeling; biophysics
The cochlear outer hair cell (OHC) is necessary for the exquisite sensitivity of mammalian hearing because of its unique electromotile property that amplifies the cochlear traveling wave (Dallos and Corey, 1991; Ruggero and Rich, 1991). The OHC has a cylindrical shape and is attached to surrounding cells only at its apical and basal ends, leaving its lateral wall free within the perilymph of the spaces of Nuel. The lateral wall has a unique trilaminate structure composed of the plasma membrane (PM), a subplasmalemmal cytoskeleton termed the cortical lattice (CL), and a membranous organelle called the subsurface cisternae (SSC) (Fig. 1). Electron microscopic studies have demonstrated the presence of many particles localized to PM (Gulley and Reese, 1977; Saito, 1983; Forge, 1991). The CL is organized in microdomains of parallel actin filaments cross-linked with spectrin (Drenckhahn et al., 1985; Flock et al., 1986; Holley and Ashmore, 1988a). Pillars run between the actin filaments and the plasma membrane (Flock et al., 1986; Arima et al., 1991).

When isolated from the organ of Corti, the electromotile response demonstrates an increasing amount of displacement with increasing distance from the point of $\mathrm{OHC}$ fixation. This pattern of displacement has been postulated to originate from multiple, evenly distributed unit motors along the lateral wall (Holley and Ashmore, 1988b; Dallos et al., 1991; Hallworth et al., 1993), termed molecular motors. In the most popular models of electro-

Received July 23, 1997; revised Oct. 9, 1997; accepted Oct. 10, 1997.

This project was supported by research grants from the Deafness Research Foundation (to J.S.O.) and Grants DC00354 and DC02775 (to W.E.B.) from the National Institute on Deafness and Other Communication Disorders. We are grateful to Drs. B. R. Alford, A. Bullen, R. A. Eatock, H. A. Jenkins, S. Popel, R. Raphael, T. Ratnanather, P. Saggau, A. Spector, and M. Zhi. N. Gavia and C. Shope provided technical support. Illustrations were done by S. Carmichael.

Correspondence should be addressed to Dr. John S. Oghalai, Bobby R. Alford Department of Otorhinolaryngology and Communicative Sciences, Baylor College of Medicine, One Baylor Plaza, Houston, TX 77030.

Copyright (C) 1997 Society for Neuroscience $\quad 0270-6474 / 97 / 180048-11 \$ 05.00 / 0$ motility, the motors are driven by the transmembrane potential of the OHC (Santos-Sacchi and Dilger, 1988) and undergo conformational changes leading to changes in lateral wall surface area (Dallos et al., 1993; Santos-Sacchi, 1993; Iwasa, 1994, 1996). The particles within the PM may represent integral membrane proteins that are the molecular motors (Santos-Sacchi, 1991; Ashmore, 1992; Iwasa and Chadwick, 1992; Kalinec et al., 1992; Iwasa, 1993; Gale and Ashmore, 1994; Kakehata and SantosSacchi, 1995, 1996).

How do motors based in the lateral wall direct their forces to change cell length? The nature of force coupling pathways among the three layers of the lateral wall is central to this question. We used the technique of fluorescence-imaged microdeformation (Discher et al., 1994; Discher and Mohandas, 1996) to evaluate stiffness and tethering within the OHC lateral wall. Di-8ANEPPS, NBD- $\mathrm{C}_{6}$-ceramide, and fluorescent conjugates of phalloidin specifically labeled the PM, SSC, and CL, respectively. Micropipette aspiration (Evans, 1973; Evans and Skalak, 1979) produced a controlled deformation of the OHC lateral wall. Brownell et al. (1995) and Sit et al. (1997) found that vesiculation occurred at high aspiration pressures, but they could not determine the composition of the vesicle. We discovered that during vesiculation the PM separated from the underlying CL and SSC. Additionally, we found that the stiffness parameter of the PM was significantly less than that of the combined CL and SSC. Hence, this report evaluates the interactions among components of the lateral wall of the OHC. Biophysical studies of these highly organized structures may help to understand the cytoskeletalplasma membrane interactions in other cell types.

\section{MATERIALS AND METHODS}

OHC isolation. Albino guinea pigs of either sex weighing 200-300 gm and having a normal startle response to a handclap were decapitated. The temporal bones were taken and the middle ear bullae opened. The otic 


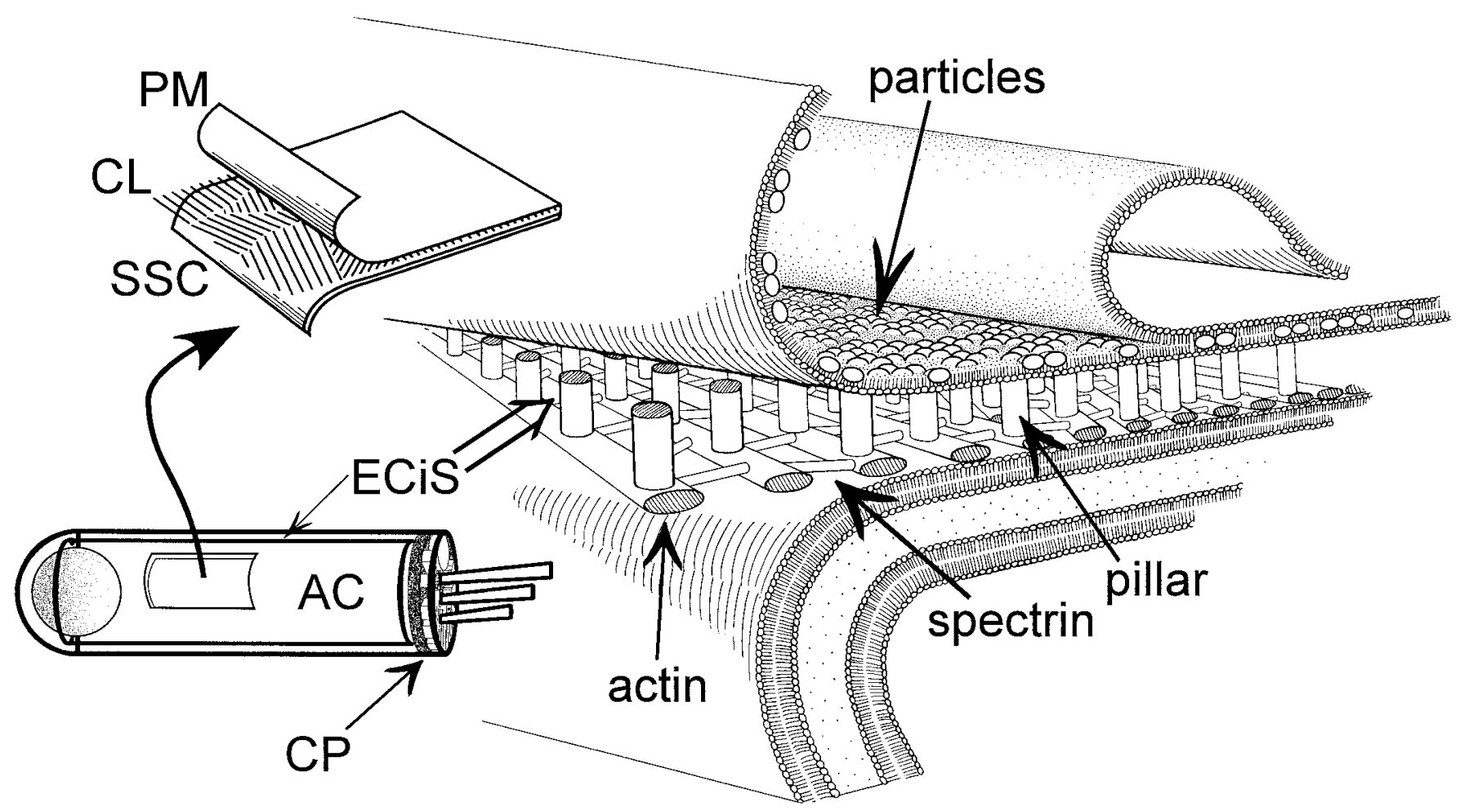

Figure 1. The outer hair cell (OHC) lateral wall. The OHC (lower left) is a cylindrical epithelial cell, with stereocilia at the apex and the nucleus at the base. The stereocilia are rooted in the cuticular plate $(C P)$. The lateral wall is a trilaminate structure composed of the plasma membrane $(P M)$, the cortical lattice $(C L)$, and the subsurface cisternae $(S S C)$. The $P M$ has been visualized by electron microscopy and noted to have a high density of membrane particles. The $C L$ is composed of microdomains of parallel actin filaments, which are cross-linked by spectrin. Although the actin orientation can vary between microdomains by $>90^{\circ}$, the average orientation is nearly circumferential, with a mean angle to the transverse axis of $9-15^{\circ}$ (Holley and Ashmore, 1988a; Holley, 1996). The $P M$ is bonded to the $C L$ via pillar molecules of unknown composition. The $S S C$ rests just inside the $C L$. The axial core $(A C)$ is the center of the cell, and the extracisternal space $(E C i S)$ is the fluid space between the SSC and the $P M$, in which lies the $C L$.

capsule was removed, and the spiral ligament was peeled off to expose the organ of Corti. The modiolus with the intact organ of Corti was removed from the temporal bone.

Two different experimental preparations were used. For OHCs that

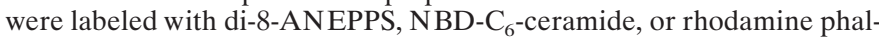
loidin, the respective staining protocols were performed in situ (described below). After staining, the organ of Corti was rinsed with extracellular solution consisting of (in mM): $135 \mathrm{NaCl}, 4 \mathrm{KCl}, 1 \mathrm{MgCl}_{2}, 2$ $\mathrm{CaCl}_{2}, 10 \mathrm{HEPES}, 10$ glucose, and $0.1 \%$ bovine serum albumin (Sigma, St. Louis, MO). The extracellular solution had an osmolality of 285-290 $\mathrm{mOsm} / \mathrm{kg}$. Mechanical trituration without enzyme was performed to dissociate the OHCs (Brownell et al., 1985). In contrast, for OHCs that were labeled with Texas Red-X phalloidin, intracellular staining was performed via tight-seal, whole-cell patch pipette dialysis (described below) after the isolation procedure. For these cells the organ of Corti was removed from the modiolus and incubated at $31^{\circ} \mathrm{C}$ for 6 min with trypsin (Type III, Sigma) at a concentration of $0.5 \mathrm{mg} / \mathrm{ml}$. Gentle pipetting was performed to dissociate the cells. The extracellular solution was L-15 (Life Technologies, Gaithersburg, MD) with 5 mM HEPES, which had an osmolality of $315-320 \mathrm{mOsm} / \mathrm{kg}$. This solution had a slightly higher osmolality, which seemed to aid the formation of tight seals. All solutions had a $\mathrm{pH}$ of 7.4 and were at room temperature $\left(22-24^{\circ} \mathrm{C}\right)$.

All OHCs were plated onto the glass bottom of an uncoated microwell Petri dish (MatTek, Ashland, MA). Isolated cells were selected for study on the basis of standard morphological criteria within $4 \mathrm{hr}$ of animal death. Under the light microscope, healthy cells display a characteristic birefringence, a uniformly cylindrical shape without regional swelling, a basally located nucleus, and no Brownian motion of subcellular cytoplasmic particles (Shehata et al., 1991).

Di-8-ANEPPS labeling. Di-8-ANEPPS is a fluorescent molecule with a nonpolar region that inserts into membranes and a polar region that is responsible for the fluorescence. This dye stains the PM of the OHC very well and does not internalize significantly (Fluhler et al., 1985). We modified the labeling techniques of Bullen et al. (1997). To make the stock solution, we dissolved di-8-ANEPPS (D-3167, Molecular Probes, Eugene, OR) in dimethyl sulfoxide (DMSO) to a concentration of 10 $\mathrm{mm}$. Aliquots were stored at $4^{\circ} \mathrm{C}$. Before use, extracellular solution was added to the aliquot, giving a final di-8-ANEPPS concentration of 150 $\mu \mathrm{M}$ and a final DMSO concentration of $1.5 \%$. The organ of Corti was incubated in this solution at room temperature for $10 \mathrm{~min}$ and then rinsed with the standard extracellular solution to remove the DMSO and unbound dye.

$\mathrm{NBD}-\mathrm{C}_{6}$-ceramide labeling. $\mathrm{NBD}-\mathrm{C}_{6}$-ceramide is a labeled precursor of sphingomyelin. In more conventional cells, it preferentially labels the Golgi apparatus (Lipsky and Pagano, 1985). In the OHC, although

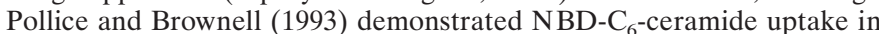
the SSC, it was unclear whether the dye was present in the PM as well.

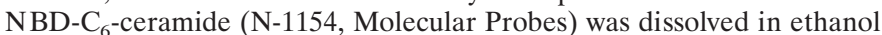
$(1 \mathrm{mg} / \mathrm{ml})$. A $2 \times$ stock solution was made by diluting the ceramide $(20$ $\mathrm{nmol} / \mathrm{ml}$ ) into extracellular solution containing defatted bovine serum albumin $\left(0.68 \mathrm{mg} / \mathrm{ml}\right.$; A-0281, Sigma). Aliquots were stored at $-20^{\circ} \mathrm{C}$. An aliquot was diluted with extracellular solution to a final concentration of $10 \mathrm{nmol} / \mathrm{ml}$ of NBD-C 6 -ceramide and $0.34 \mathrm{mg} / \mathrm{ml}$ defatted bovine serum albumin. The organ of Corti was incubated in this solution at room temperature for $30 \mathrm{~min}$ and then rinsed with the standard extracellular solution.

Rhodamine phalloidin labeling. Phalloidins bond specifically to F-actin, and fluorescent conjugates have been shown to label the CL in the OHCs (Carlisle et al., 1988; Slepecky, 1989). Because phalloidins are hydrophilic, introducing the dye into the intracellular compartment is difficult in living cells. One method we used was to create pores in the plasma membrane before a bath application of dye. One vial (300 U) of rhodamine phalloidin (R-415, Molecular Probes) was dissolved in $1.5 \mathrm{ml}$ of methanol. Aliquots were dried and stored at $-20^{\circ} \mathrm{C}$. The $\mathrm{OHC} \mathrm{PM}$ was lightly permeabilized with $0.05 \%$ saponin (S-2149, Sigma) for $5 \mathrm{~min}$. The organ of Corti was incubated in a final rhodamine phalloidin concentra- 
Figure 2. The micropipette aspiration technique. This technique involves bringing a glass pipette with an internal diameter of $\sim 3 \mu \mathrm{m}$ in contact with the lateral wall of the cell. As negative pressure is applied, a tongue of lateral wall is pulled inside the pipette. The tongue length is measured at different aspiration pressures and used to determine the stiffness parameter (see Fig. 7). With increasing suction pressure, the individual components of the lateral wall can be visualized (see Fig. 8).
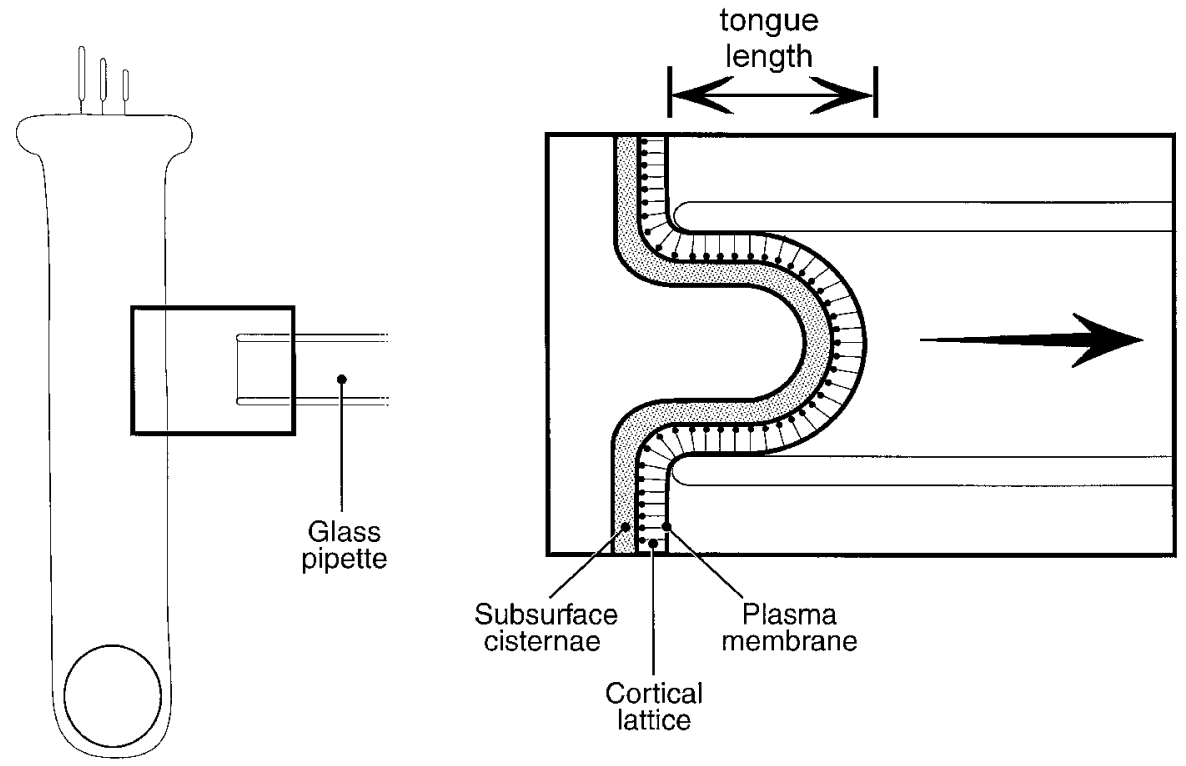

tion of $100 \mathrm{U} / \mathrm{ml}$ for $30 \mathrm{~min}$ at room temperature and then rinsed with the standard extracellular solution.

Texas Red-X phalloidin labeling. To stain actin while maintaining the integrity of the PM (in contrast to the rhodamine phalloidin staining procedure), we also performed an intracellular dialysis technique, using a tight-seal whole-cell ruptured patch configuration. One vial (300 U) of Texas Red-X phalloidin (T-7471, Molecular Probes) was dissolved in 100 $\mu \mathrm{l}$ of methanol. Aliquots were dried and stored at $-20^{\circ} \mathrm{C}$. The patch pipette intracellular solution consisted of (in $\mathrm{mm}$ ): $150 \mathrm{KCl}, 5 \mathrm{MgCl}_{2}, 5$ EGTA, and 10 HEPES, as well as $60 \mathrm{U} / \mathrm{ml}$ Texas Red-X phalloidin. The intracellular solution had an osmolality of $305-310 \mathrm{mOsm} / \mathrm{kg}$ and a $\mathrm{pH}$ of 7.4 and was at room temperature. Patch pipettes were fabricated from borosilicate thin wall capillary tubing (GC150T-10, Warner Instrument, Hamden, CT), using a two-stage vertical pipette puller (PP-83, Narishige, Tokyo, Japan). The tips were fire-polished to an internal diameter of $\sim 1.5 \mu \mathrm{m}$ (MF-83, Narishige), with a final impedance of 2-4 M $\Omega$. Seals were formed at the base of the OHC by using an Axopatch 200B amplifier and pCLAMP6 software (Axon Instruments, Foster City, CA). The cells were held at their zero current potential; only cells with potentials negative to $-50 \mathrm{mV}$ were used.

Confocal microscopy. Data were gathered by a scanning confocal system (MRC-600, Bio-Rad, Hercules, CA) with a krypton/argon laser, configured on an Axiovert 35 microscope (Zeiss, Oberkochen, Germany). Images were obtained with a $100 \times$ oil immersion objective (numerical aperture 1.30; Plan-NEOFLUAR, Zeiss). Fluorescent images of cells labeled with di-8-ANEPPS and NBD-C 6 -ceramide were collected, using an excitation wavelength of $488 \mathrm{~nm}$. Images of cells labeled with fluorescent phalloidins were collected at an excitation wavelength of 568 $\mathrm{nm}$. Emission wavelengths were collected, using long-pass filters with corner wavelengths of $515 \mathrm{~nm}$ for the membrane dyes and 585 for the actin dyes. Images equivalent to those normally seen by light microscopy were collected with the transmitted light path of the system, using Nomarski optics. In Figure $5 d$, the fluorescent signal was so low that we had to open the confocal aperture all the way to visualize the dye staining. This greatly increased the $z$-plane thickness. In all other confocal images a more typical aperture setting was used, and the thickness of the $z$-plane sections was $\sim 1.0 \mu \mathrm{m}$.

Micropipette aspiration and stiffness parameter calculations. The micropipette aspiration technique (Fig. 2) was used to produce a controlled deformation of the lateral wall of the OHC. Aspiration micropipettes with internal diameters of $\sim 3 \mu \mathrm{m}$ were fabricated from borosilicate glass capillary tubes (LG16; outer diameter, $1.65 \mathrm{~mm}$, inner diameter, $1.1 \mathrm{~mm}$; Dagan, Minneapolis, MN) with an automated glass pipette puller (BB$\mathrm{CH}-\mathrm{PC}$, Switzerland) and microforge (Microforge De Fonbrune, Aloe Scientific, St. Louis, MO). The micropipette was connected to a water column via polyethylene tubing, and both were filled with extracellular solution. The micropipette was mounted on a joystick-controlled electronic micromanipulator (Zeiss) and advanced to the center of the field of view. The level of the water column was referenced to the microscope stage (i.e., the zero pressure point).
Once a healthy cell was located, the micropipette was brought into contact with the midlateral wall of the OHC. Negative pressure was applied in a stepwise manner as measured from the null point. This was performed by sequentially decreasing the pressure by $1 \mathrm{~cm}$ of $\mathrm{H}_{2} \mathrm{O}(1 \mathrm{~cm}$ of $\mathrm{H}_{2} \mathrm{O}=0.09807 \mathrm{nN} / \mu \mathrm{m}^{2}$ ) and then waiting $30 \mathrm{sec}$. This was repeated until $-20 \mathrm{~cm}$ of $\mathrm{H}_{2} \mathrm{O}$ was reached, the cell burst, or vesiculation occurred. Tongue lengths were measured from the digitized transmitted and fluoto the tip of the micropipette. The resolution of the digitized images was 5.5 pixels $/ \mu \mathrm{m}$. Early on, we discovered that tongue length varies with the internal diameter of the pipette, such that larger pipettes tended to produce longer tongues before vesiculation occurred. To minimize this variability, we used only pipettes of similar diameter to collect the stiffness data presented in this report (inner diameter, $2.88 \pm 0.34 \mu \mathrm{m}$; mean $\pm \mathrm{SD} ; n=32$ ). Because the aspiration pipette was held at an angle to the stage, the tongue length we measured was a projection of the true length. However, this effect was minimized by keeping the angle of approach shallow $\left(<20^{\circ}\right)$ and consistent for all experiments.

The stiffness parameter $\left(S_{P}\right)$ is calculated from the tongue length versus aspiration pressure data (Evans, 1973; Chien et al., 1978; Spector et al., 1996, 1997a; Sit et al., 1997). By applying a controlled amount of pressure to the wall of a cell, we can measure the amount of subsequent bending, and we can calculate $S_{P}$ to quantify the deformability of the lateral wall. $S_{P}$ is defined as:

$$
S_{p}=\frac{-\Delta P^{*} r}{\Delta L_{t} / r}=\frac{-\Delta P^{*} r^{2}}{\Delta L_{t}}=\text { constant } *\left[\frac{\Delta \text { Force }}{\Delta \text { Deformation }}\right],
$$

where $\Delta P$ is the change in aspiration pressure applied to the lateral wall through the micropipette, $\Delta L_{t}$ is the change in tongue length, and $r$ is the internal radius of the micropipette. Hence, $S_{P}$ is $1 /$ slope of plots of experimental data (see Fig. 7) and is proportional to the change in force per change in deformation. We calculated $S_{P}$ for unlabeled controls and for cells labeled with the membrane dyes.

\section{RESULTS}

\section{Labeling of the plasma membrane}

Figure 3 shows an OHC stained with di-8-ANEPPS under lowpressure deformation. The transmitted image (Fig. $3 a$ ) shows the lateral wall tongue being aspirated into the micropipette. The apex of the cell was out of the plane of focus. The fluorescent image (Fig. $3 b)$ demonstrates labeling of the basolateral surfaces of the cell. Di-8-ANEPPS also stained the apex and stereocilia (data not shown). There was no staining of intracellular structures. The tongue lengths in both images are the same; note that the tip of the tongue has a rounded shape. With higher aspiration pressures, the tongue length increases until the most distal portion detaches from rescent images, from the apex of the aspirated portion of the lateral wall 


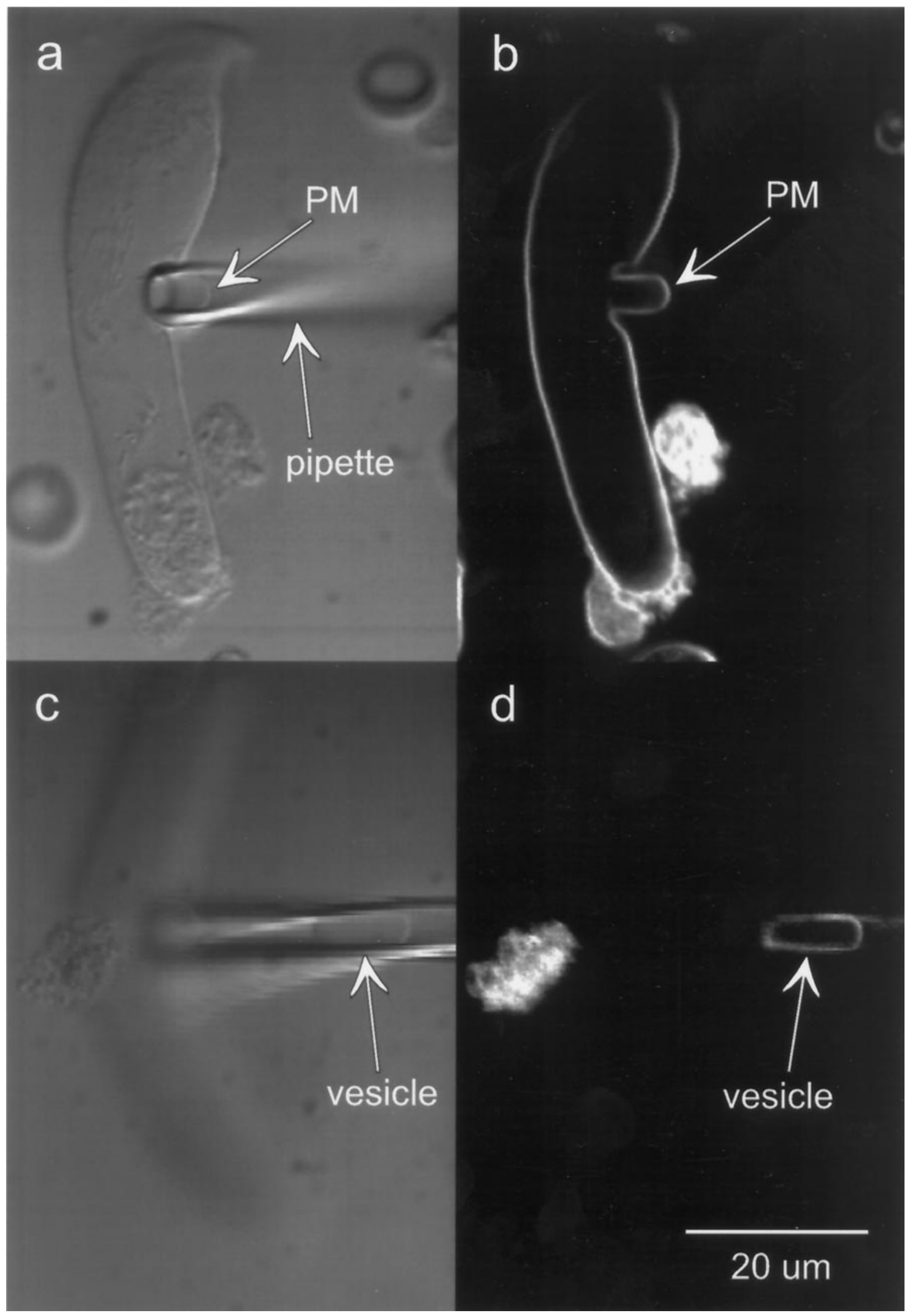

Figure 3. Di-8-ANEPPS labeling of the PM. $a$, Transmitted image of an OHC undergoing micropipette aspiration $\left(-11 \mathrm{~cm}^{-} \mathrm{H}_{2} 0\right) . b$, The simultaneous fluorescent image of $a$ demonstrates staining of the $P M$. The apex of the cell is out of the focal plane in this image; however, the stereocilia were always labeled. $c$, Transmitted image of a vesicle that was released as the suction pressure was increased $\left(-15 \mathrm{~cm}\right.$ of $\left.\mathrm{H}_{2} 0\right)$. $d$, The fluorescent image shows that the vesicle contains PM. 
the cell, forming a vesicle. An example of a vesicle is demonstrated in Figure $3 c$. Its fluorescent image (Fig. $3 d$ ) demonstrates that the wall of the vesicle is labeled. Because di-8-ANEPPS is known to label the PM, the PM is a component of the vesicle $(n=10)$.

\section{Labeling of the subsurface cisternae}

Figure 4 demonstrates an $\mathrm{OHC}$ that was labeled with $\mathrm{NBD}-\mathrm{C}_{6}$ ceramide. The transmitted image (Fig. $4 a$ ) shows the tongue of lateral wall aspirated inside the micropipette. Two layers of the lateral wall are visible within the pipette: the outer layer is indicated PM, and the inner layer is indicated SSC. The fluorescent image (Fig. 4b) shows labeling of the lateral wall and of some intracellular structures, including the nuclear membrane. The length of the tongue in Figure $4 b$ corresponds to that of the inner layer in Figure $4 a$. This figure demonstrates that this $\mathrm{NBD}-\mathrm{C}_{6}$ ceramide staining procedure labels the SSC, but not the PM. This image also shows that the PM can be separated from the SSC. After separation an inner tongue could be seen only in the transmitted image in approximately one-half of the cells; however, by fluorescence labeling, an inner tongue was visible in every cell. The SSC tongue in the fluorescent image has a flat tip, as opposed to the round tip of the PM tongue in the transmitted image. Figure $4 c$ is the transmitted image of a vesicle pulled from a NBD-C ${ }_{6}$ ceramide-stained cell. Because the simultaneous fluorescent image (Fig. 4d) does not show labeling of the vesicle, SSC membranes are not a component of the vesicle $(n=11)$.

\section{Labeling of the cortical lattice}

Figure 5, $a$ and $b$, demonstrates a cell that was permeabilized with saponin and subsequently labeled for F-actin with rhodamine phalloidin. The transmitted image (Fig. $5 a$ ) shows the poor condition of the cell, which is to be expected after treatment with saponin. However, a tongue of the lateral wall could be aspirated into the pipette. The fluorescent image (Fig. $5 b$ ) shows a large amount of labeling at the apex as well as faint labeling along the lateral walls. This labeling pattern demonstrates the difference in the density of F-actin in the cuticular plate and stereocilia versus the CL. The labeled tongue is visible and is at the same location as the tip of the tongue seen in the transmitted image. Aspiration pressure was increased in these cells until they ruptured (typically -20 to -30 $\mathrm{cm}$ of $\mathrm{H}_{2} \mathrm{O}$ ). No separation of the PM or vesiculation was ever noted $(n=10)$, most likely because of permeabilization of the PM.

Figure 5, $c$ and $d$, demonstrates a cell that was labeled intracellularly for F-actin with Texas Red-X phalloidin. This technique did not require the use of saponin, so the integrity of the PM was preserved. Simultaneous aspiration and patch pipettes were used. First, aspiration of the lateral wall was performed to hold a tongue at constant pressure, usually approximately $-10 \mathrm{~cm}$ of $\mathrm{H}_{2} \mathrm{O}$. Then, a whole-cell configuration was achieved to introduce the dye inside the OHC. It took $\sim 10-15 \mathrm{~min}$ after entering whole-cell configuration for sufficient dye to enter the OHC so that imaging could be performed. The current-to-voltage relationships of the $\mathrm{OHC}$, as monitored by periodic voltage-clamp step protocols, did not change during the staining process (data not shown). The transmitted image (Fig. 5c) shows a tongue of the lateral wall being aspirated into the pipette, with two visible layers. The patch pipette was at the base of the cell and is not visible in this image. The fluorescent image (Fig. $5 d$ ) shows strong labeling of the cuticular plate and faint labeling along the lateral walls, demonstrating the CL. The fluorescence-labeled tongue is at the same location as the inner tongue seen in the transmitted image. Because the dye staining by intracellular dialysis is quite weak, we had to open the aperture completely on the confocal microscope. The resulting thick depth of field explains why the fluorescence in the tongue appears confluent rather than distributed along the perimeter as in the previous images. However, this figure demonstrates that separation of the PM involves peeling it away not only from the SSC (as in Fig. 4) but from the CL as well $(n=3)$. So an inner layer visible by fluorescent phalloidin labeling, $\mathrm{NBD}-\mathrm{C}_{6}$-ceramide labeling, or by transmitted image represents the $\mathrm{CL}$ and the SSC. The PM is represented by the outer layer. We term the combination of the two structures that make up the inner layer the CL/SSC complex.

\section{Dual labeling of the plasma membrane and the subsurface cisternae}

The cell in Figure 6 was stained with both di-8-ANEPPS and NBD-C 6 -ceramide. A series of fluorescent images demonstrates the sequence of separation and vesiculation. In Figure $6 a$, the PM and CL/SSC complex were indistinguishable from each other. The layers were bonded together, and both had a round tip. The lines radiating from the base of the tongue are folds in the cell membranes caused by the applied suction pressure. They disappeared as the manipulation progressed (Fig. $6 b-f$ ), perhaps because of an increase in cell turgor pressure. These folds were not visible in Figures 3-5. In Figure 6b, the PM separated from the CL/SSC complex. At this point the PM continued to have a round tip, whereas the tip of the SSC flattened out. In Figure $6 c-e$, the PM tongue continued to elongate, whereas the SSC tongue remained in nearly the same location. Finally, in Figure $6 f$, the PM tongue broke free to form a vesicle, leaving its base to reseal. This process can continue, leading to the release of multiple vesicles. For example, we have aspirated up to 26 vesicles from a single cell.

\section{Stiffness parameters}

Both PM and CL/SSC complex tongue lengths were measured from OHCs stained with di-8-ANEPPS and NBD-C -ceramide $_{6}$ as the aspiration pressure was increased. All data points from 11 cells are plotted (Fig. 7). The dotted and solid lines represent the PM and the CL/SSC complex, respectively, both before and after separation. The reciprocal of the slope is the $S_{P}$ of the structure. These lines are the average of the slopes and $y$-intercepts of lines fit to each of the data points of the 11 cells. The scatter apparent in the figure is attributable to variation in the $y$-intercept points (i.e., the starting tongue lengths) of different cells; however, the slope $\left(1 / S_{P}\right)$ varied little (see data below). The inset is an example of the data recorded from one cell.

The PM and CL/SSC complex overlapped with low aspiration pressures. The average $S_{P}$ of the intact trilaminate wall was $0.79 \pm 0.18 \mathrm{nN} / \mu \mathrm{m}($ mean $\pm \mathrm{SD})$. The point of separation had an $x$ value of $1.67 \pm 0.30 \mathrm{nN} / \mu \mathrm{m}$ (mean $\pm \mathrm{SD})$, corresponding to a pressure of $-1.20 \pm 0.23 \mathrm{nN} / \mu \mathrm{m}^{2}$ (mean $\pm \mathrm{SD}$ ), and is represented by the large circle on the graph. After this point the tongue length of the PM increased more rapidly as the pressure was increased. Its $S_{P}$ was $0.17 \pm 0.08 \mathrm{nN} / \mu \mathrm{m}$ (mean $\pm \mathrm{SD}$ ), which is significantly different from that of the trilaminate wall (paired Student's $t$ test; $p<0.001)$.

On the other hand, the CL/SSC complex tongue length did not increase as rapidly with pressure increments after separation as it did before separation. Although the PM tongue maintained a round shape throughout the aspiration process, the flat tips of the CL and SSC tongues after separation (more clearly seen in Figs. 4b, $5 d$ ) imply that there was no pressure gradient across them. So, after separation, the negative pressure applied by the micropipette acted 


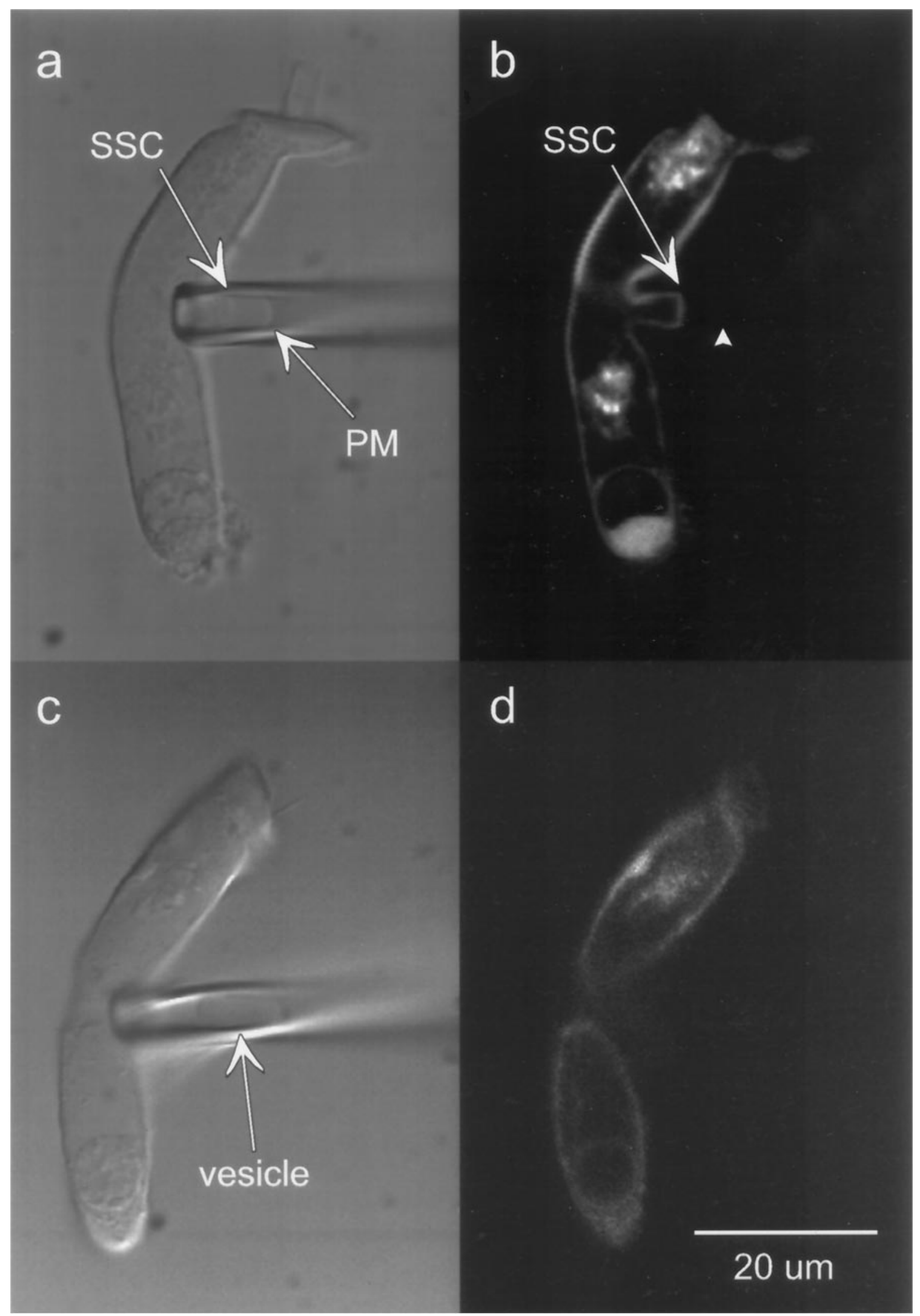

Figure 4. NBD-C $\mathrm{C}_{6}$-ceramide labeling of the SSC. $a$, Transmitted image of an $\mathrm{OHC}$ undergoing micropipette aspiration $\left(-15 \mathrm{~cm}\right.$ of $\left.\mathrm{H}_{2} 0\right)$. Note that for this cell both an outer layer $(P M)$ and an inner layer $(S S C)$ are visible in the lateral wall tongue. The longitudinal bending of the cell is caused by the aspiration

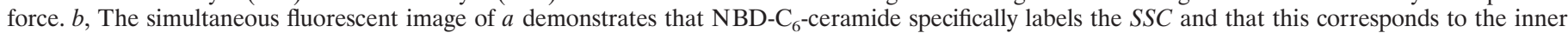
layer seen in the transmitted image. The PM was not labeled; however, its position is marked by the arrowhead. The stereocilia were never labeled. $c$, Transmitted image of a vesicle that was released $\left(-17 \mathrm{~cm}\right.$ of $\left.\mathrm{H}_{2} 0\right) . d$, The fluorescent image shows that the SSC is not a component of the vesicle, because it does not contain label. 


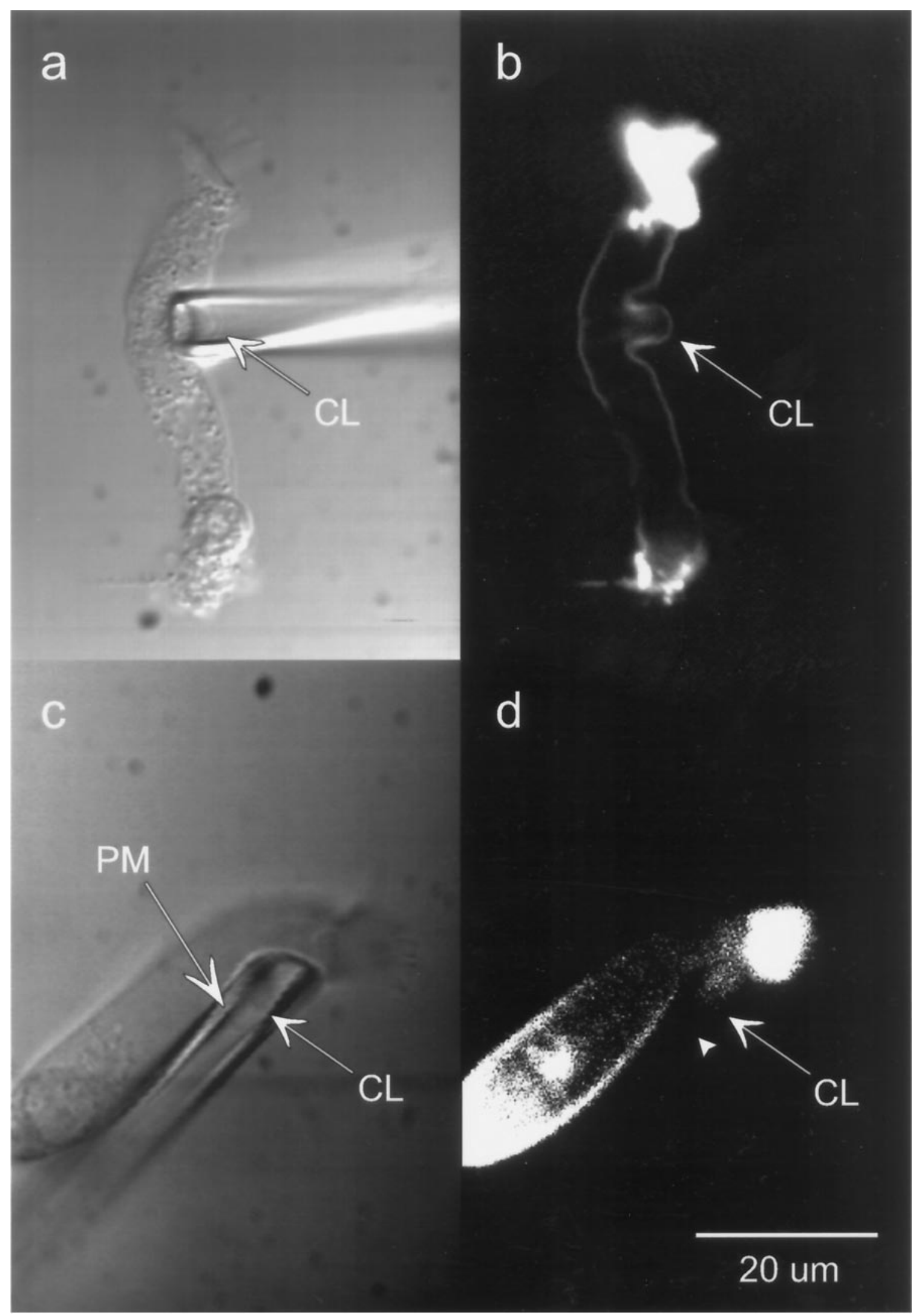

Figure 5. Fluorescent phalloidin labeling of the CL. $a$, Transmitted image of an OHC after treatment with saponin and staining with rhodamine phalloidin $\left(-10 \mathrm{~cm}\right.$ of $\left.\mathrm{H}_{2} 0\right)$. Note that a tongue could be pulled even with the poor condition of the cell. No vesicles could be formed $(n=10)$. $b$, The fluorescent image demonstrates strong labeling of actin within the stereocilia and cuticular plate. The weaker labeling along the lateral walls corresponds to staining of actin within the $C L$ (arrow). $c$, Transmitted image of a cell undergoing aspiration $\left(-10 \mathrm{~cm}^{\circ} \mathrm{H}_{2} 0\right)$. Note the two layers visible in the tongue. The cell was also whole-cell-patched at the base to introduce Texas Red-X phalloidin; however, the patch pipette is not (Figure legend continues) 

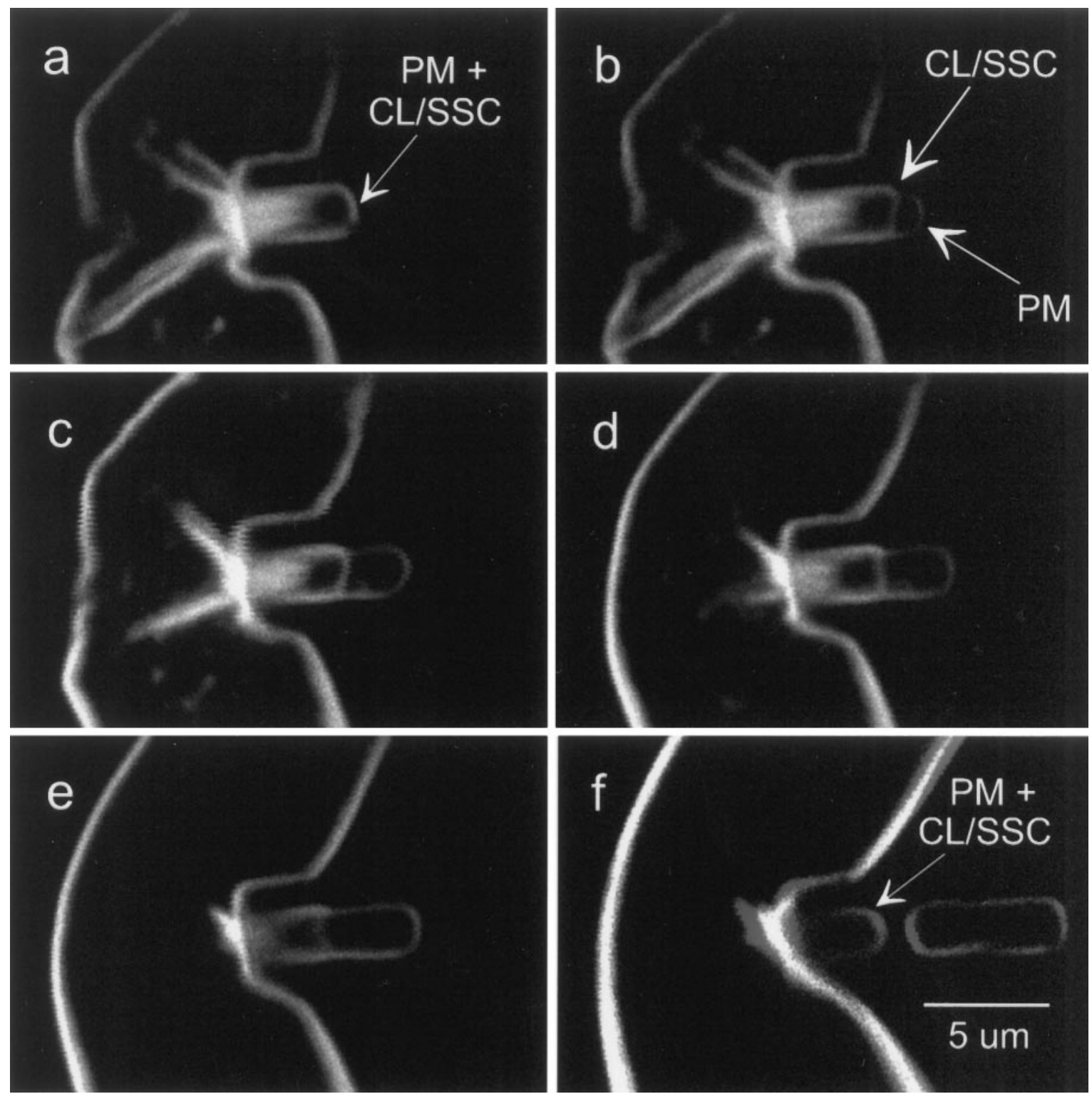

Figure 6. Dual labeling of the PM and SSC. This figure captures the sequence of separation and vesiculation during micropipette aspiration of the OHC lateral wall. $a$, The $P M$ and the $S S C$ overlapped initially $\left(-10 \mathrm{~cm}\right.$ of $\left.\mathrm{H}_{2} 0\right) . b$, The $P M$ separated from the $S S C\left(-14 \mathrm{~cm}\right.$ of $\left.\mathrm{H}_{2} 0\right)$. $c-e$, The PM tongue elongated, whereas the SSC tongue stayed nearly the same length $\left(-14 \mathrm{~cm}\right.$ of $\left.\mathrm{H}_{2} 0\right)$. $f$, The PM vesiculated and then resealed over the SSC ( $-14 \mathrm{~cm}$ of $\mathrm{H}_{2} 0$ ). To enhance visualization because of the angle of the pipette to the microscope stage, we created this image by summing three sequential images taken at slightly different $z$-plane levels. Previous images $(a-e)$ were single $z$-plane levels.

to pull directly on the PM alone. This means that the reciprocal of the slope of the line indicating the CL/SSC complex (Fig. 7) is not representative of its $S_{P}$. Hence, subtraction of the $S_{P}$ of the PM from the $S_{P}$ of the trilaminate wall was performed to determine this value $(0.79-0.17=0.62 \mathrm{nN} / \mu \mathrm{m})$. So the $\mathrm{PM}$ is responsible for $22 \%$ of the $S_{P}(0.17 / 0.79)$ of the trilaminate wall, and the remaining two layers comprise the remaining $78 \%(0.62 / 0.79)$. The lower $S_{P}$ of the $\mathrm{PM}$ indicates that it is more compliant than the CL/SSC complex.

To verify that the dyes did not affect lateral wall mechanics, we compared $S_{P}$ and separation pressures of labeled cells versus con- trols. $S_{P}$ of unlabeled controls $(n=11)$, as well as OHCs stained with di-8-ANEPPS $(n=10)$ or NBD-C 6 -ceramide $(n=11)$, were not significantly different $(p>0.1)$ both before and after separation (data not shown). Additionally, the separation pressures were not significantly different ( $p>0.1$; data not shown).

\section{DISCUSSION}

\section{Selective labeling and the extracisternal space}

Previous reports have demonstrated fluorescent labeling of structures within the OHC lateral wall (Carlisle et al., 1988; Slepecky,

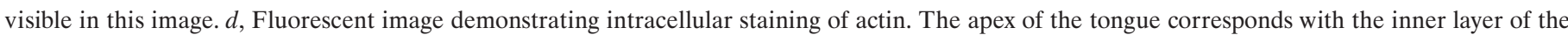

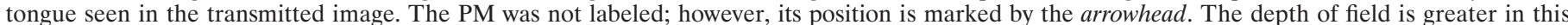

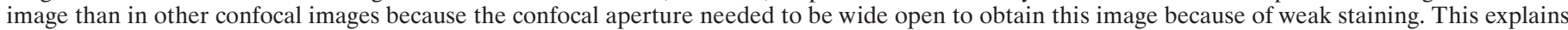
why a discrete line of staining along the periphery is not present, as in $b$. 
Figure 7. Tongue length versus aspiration pressure. These data were taken from dual-labeled OHCs $(n=$ 11). The circles represent PM tongue lengths, and the triangles represent SSC tongue lengths. The point of separation is represented by the large circle. The dotted and solid lines correspond to the PM and CL/SSC complex, respectively. The four lines were created by averaging the slopes and $y$-intercepts of linear fits to each of the 11 cells both before and after the point of separation. The corresponding stiffness parameters $\left(S_{P}\right)$ are the reciprocal of the slopes of each line and are defined in units of $\mathrm{nN} / \mu \mathrm{m}$. The inset is an example of data taken from one cell.

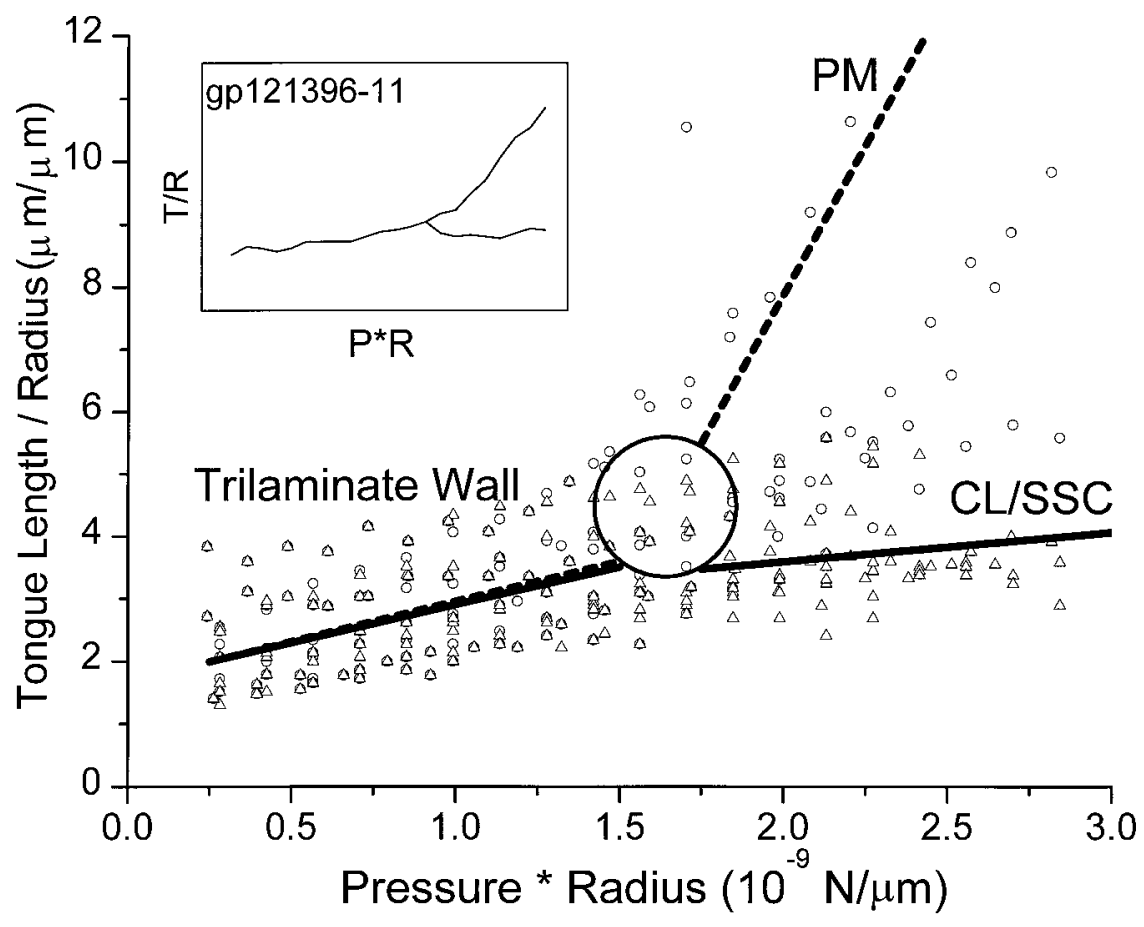

1989; Holley et al., 1992; Ikeda and Takasaka, 1993; Pollice and Brownell, 1993; Raphael et al., 1994); however, this is the first report clearly demonstrating selective staining of each of the three layers that make up the OHC lateral wall in a living, freshly dissociated preparation. The cells appeared healthy by microscopy, remaining viable for up to $4 \mathrm{hr}$ after animal death, similar to unlabeled isolated OHCs. The Texas Red-X phalloidin labeled cells had current-to-voltage relationships that appeared similar to previous reports (Nakagawa et al., 1994a,b). Also, di-8-ANEPPS has been shown previously not to affect the OHC current-to-voltage relationships (Nakagawa et al., 1997). The NBD-C 6 -ceramide staining procedure only labels the SSC, which is consistent with the absence of label in the stereocilia.

With the application of negative pressure, a tongue of lateral wall was pulled into the micropipette. Initially, the trilaminate structure was bonded together. As the suction pressure was increased, the PM separated from the lateral wall, representing disruption of the tethering between the PM and the CL/SSC complex. The fluid compartment between the two layers is the extracisternal space. Not only were we able to determine that the vesicle is composed of PM and does not contain actin from the CL or membranes from the SSC, but we could further dissect their structural contribution to wall mechanics by quantitating their deformation.

\section{Component contributions to lateral wall stiffness}

Our findings can be compared with other experiments in which the stiffness of the lateral wall has been altered. Although the absolute values of stiffness measurements may vary, depending on the type of experimental deformation (Spector et al., 1997b), comparison of the relative stiffnesses between lateral wall components can be performed safely. Kalinec et al. (1992) found that, after intracellular perfusion of trypsin in OHCs to disrupt the CL and SSC, the normal cylindrical shape was altered greatly such that it became more spherical, without lateral wall stiffness to counteract turgor pressure. Although the actions of trypsin on the stiffness of the PM are unclear, this may indicate that the PM is not as stiff as the CL/SSC complex. When the complimentary manipulation was performed, i.e., when OHCs were treated with Triton X-100 to remove the PM (Holley and Ashmore, 1988a) (E. Chan, A. Suneson, and M. Ulfendahl, unpublished results), the CL shell lengthened yet maintained its cylindrical shape, consistent with an intrinsic rigidity and shape inherent to the CL. Both of these findings support our conclusion that the CL/SSC complex is primarily responsible for lateral wall stiffness.

However, our observation that the lateral wall stiffness is dominated by the CL/SSC complex contrasts with the conclusions of Holley and Ashmore (1988a). They found that it took significantly more force to compress the healthy cell than the demembranated cell (i.e., the CL shell) in the longitudinal direction. They stated that this meant the majority of lateral wall stiffness is inherent to the PM. Tolomeo et al. (1996) also used the technique of Triton X-100 demembranization to determine the stiffness of the CL, with similar conclusions. However, because there would be no turgor pressure in a demembranated cell, a much lower stiffness measurement is hardly surprising. In contrast to these reports, we were able to compare $S_{P}$ of the PM with that of the CL/SSC complex in healthy cells.

\section{The role of the cytoskeleton and pillars}

Figure 8 is a representative diagram of the separation process. Although the PM can be detached from the lateral wall, the CL and SSC remain behind and are primarily responsible for the $S_{P}$ of the lateral wall. The phenomenon of separation proves that the PM is tethered to the underlying lateral wall and that this tethering can be broken. $S_{P}$ of the OHC trilaminate lateral wall is anywhere from 2 to 76 times higher than in other cells (Sit et al., 1997). There is no cell that has been documented to have a higher $S_{P}$. The cytoskeleton of most eukaryotic cells contains F-actin, tubulin, and intermediate filaments; however, the high stiffness of the OHC lateral wall may be attributable to the anisotropic nature of the $\mathrm{OHC}$ cytoskeleton, which consists of microdomains that tend to orient the actin filaments circumferentially. Interestingly, other types of hair cells (which do not have electromotility) do not appear to have 

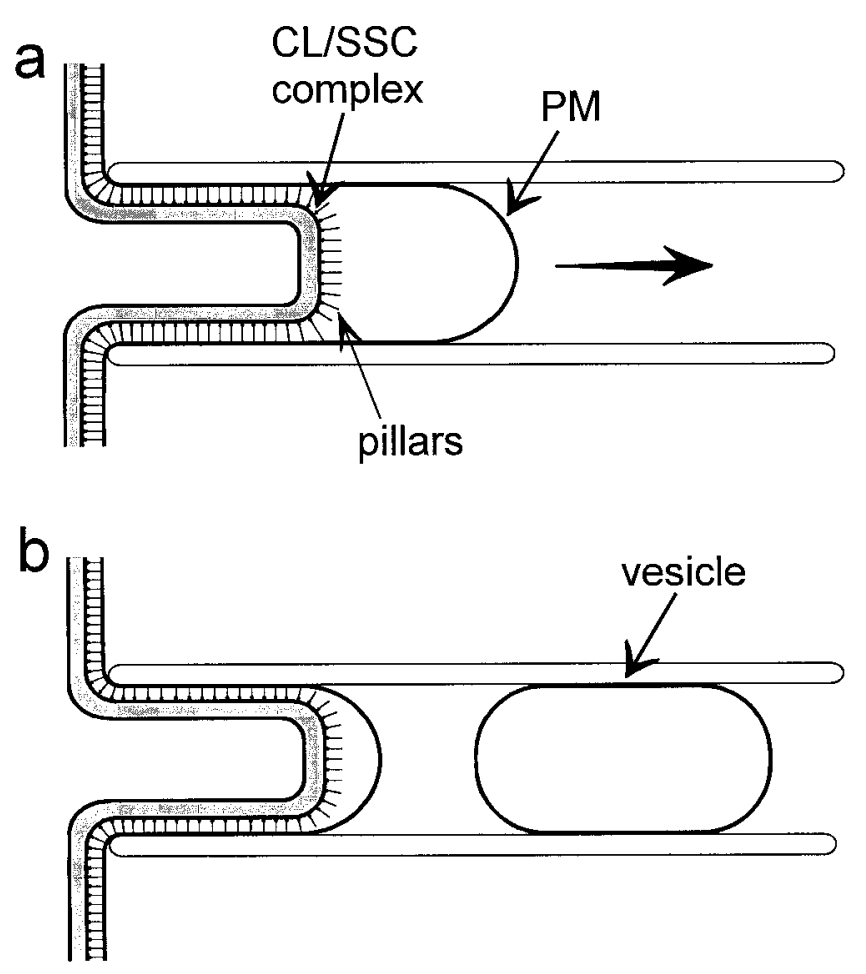

Figure 8. The phenomenon of separation. Initially, the bonded trilaminate lateral wall is pulled into the pipette (as seen in Fig. 2). After separation $(a)$, the tip of the CL/SSC complex flattens out, whereas the PM continues to elongate. $b$, The vesicle consists of only PM and does not contain actin from the $\mathrm{CL}$ or membranes from the SSC. The remaining PM reseals over the lateral wall and begins to elongate again. Although represented as remaining with the actin of the $\mathrm{CL}$ in this diagram, the actual fate of the pillars is unknown (see Discussion).

this unique structure. Additionally, similar to the $\mathrm{OHC}$, the cytoskeleton of red blood cells also contains actin and spectrin in a subplasmalemmal configuration. However, the red blood cell is orthotropic and flexible (Discher et al., 1994; Discher and Mohandas, 1996), allowing the cells to deform while passing through thin capillaries. So, with the uniquely high stiffness inherent to the $\mathrm{OHC}$ lateral wall, the pressures at which separation occur are in all likelihood above normal physiological stress conditions. This would imply that during normal electromotility in vivo the PM is tightly bound to the underlying lateral wall.

The pillars are probably responsible for this tethering. The maximal tethering force depends both on their longitudinal strength as well as on their anchoring strength into the PM and the CL (Spector et al., 1996). However, there is no clear information as to which link is broken during the aspiration experiments. In Figure 8, the pillars were shown as remaining bonded to the CL after separation, although we have no evidence to support this.

\section{Implications for electromotile force coupling}

One hypothesis to explain $\mathrm{OHC}$ force coupling is the cytoskeletal spring model proposed by Holley and Ashmore (1990). In this model the molecular motors rest within the PM, and the CL is less stiff than the PM. When the motors change in surface area, they push against themselves and their surrounding phospholipids, summating their forces and creating cell length changes. Hence, by this model, force coupling occurs within the PM because the PM has a high stiffness. An alternative possibility, suggested by Iwasa (1994) and further discussed by Tolomeo et al. (1996), is that motors in the PM transmit their force down the pillars to the CL, which then orients the forces. Because an equal and opposite force would be required to hold the motors in place within the PM, the PM must be stiffer than the CL in this theory as well.

Our findings do not support these hypotheses. Because the PM appears to be more compliant than the CL, it would seem that membrane-based motors should just push the bilayer phospholipids out of the way as they change conformation, rather than directing their force purposefully. This makes it unlikely that the motors exert a force when increasing in surface area. However, it is possible that they could still exert a force when decreasing in surface area, by pulling on the lipid bilayer. A contractile force within the PM probably could lead to changes in cell shape, because the PM can withstand the pulling force of micropipette aspiration to the point at which bending of the entire cell occurs (Figs. 3, 4; see also Sit et al., 1997). This means that, although the PM stiffness is less than the CL stiffness, the PM tensile strength is greater than CL stiffness. So if some other source of extensile force existed within the $\mathrm{OHC}$, then the mechanism of electromotility may occur via opposing contractile and extensile forces.

This extensile force might be passive resting tension from within the framework of the CL, associated with cell turgor pressure (Brownell et al., 1985; Holley and Ashmore, 1988a,b, 1990; Brownell, 1990; Shehata et al., 1991). With tethering, the pillars would distribute the total extensile force over the surface area of the lateral wall. The concept of tethering is consistent with the work of Dallos et al. (1991). Our concept of OHC electromotility is a further refinement of the model described by Santos-Sacchi (1993), and we suggest that without some type of intrinsic extensile force within the cell there could be little force coupling between the motors.

Interestingly, although the pillars clearly are tethered to the PM, the motors do not have to be fixed in place. This leaves the intriguing possibility that the motors could be mobile within the PM lipid bilayer (as in the fluid mosaic model), sensing transmembrane potential and changing in surface area correspondingly. Meanwhile, passive tension, acting to elongate the OHC, "fits" cell length to the available amount of PM surface area. There are many more particles in the PM (the postulated molecular motors) than pillars (Forge, 1991). If pillars were needed to transmit the force generated by these motors, structural connections between the motors and the pillars would be necessary. However, there have been no visualized organizations or connections between the particles and the pillars by ultrastructural studies. This discrepancy further supports the hypothesis that the motors randomly diffuse throughout the OHC lateral wall PM. Their fluidity may be limited by interactions with each other or the lipid components of the membrane bilayer. One consequence of identifying membranespecific labels is that membrane fluidity can be quantified by using fluorescence recovery after photobleaching techniques (our unpublished data).

\section{REFERENCES}

Arima T, Kuraoka A, Toriya R, Shibata Y, Uemura T (1991) Quickfreeze, deep-etch visualisation of the "cytoskeletal spring" of cochlear outer hair cells. Cell Tissue Res 263:91-97.

Ashmore JF (1992) Mammalian hearing and the cellular mechanisms of the cochlear amplifier. In: Sensory transduction (Corey DP, Roper SD, eds), pp 396-412. New York: Rockefeller UP.

Brownell WE (1990) Outer hair cell electromotility and otoacoustic emissions. Ear Hear 11:82-92.

Brownell WE, Bader CR, Bertrand D, de Ribaupierre Y (1985) Evoked mechanical responses of isolated cochlear outer hair cells. Science 227:194-196. 
Brownell WE, Ratnanather JT, Popel AS, Zhi M, Sit PS (1995) Labyrinthine lateral walls: cochlear outer hair cell permeability and mechanics. In: Active hearing (Flock A, Ottoson D, Ulfendahl M, eds), pp 167-179. Oxford, UK: Pergamon.

Bullen A, Patel SS, Saggau P (1997) High-speed, random-access fluorescence microscopy. I. High resolution optical recording with voltagesensitive dyes and ion indicators. Biophys J 73:477-491.

Carlisle L, Zajic G, Altschuler RA, Schacht J, Thorne PR (1988) Species differences in the distribution of infracuticular F-actin in outer hair cells of the cochlea. Hear Res 33:201-206.

Chien S, Sung KL, Skalak R, Usami S, Tozeren A (1978) Theoretical and experimental studies on viscoelastic properties of erythrocyte membrane. Biophys J 24:463-487.

Dallos P, Corey ME (1991) The role of outer hair cell motility in cochlear tuning. Curr Opin Neurobiol 1:215-220.

Dallos P, Evans BN, Hallworth R (1991) Nature of the motor element in electrokinetic shape changes of cochlear outer hair cells. Nature 350:155-157.

Dallos P, Hallworth R, Evans BN (1993) Theory of electrically driven shape changes of cochlear outer hair cells. J Neurophysiol 70:299-323.

Discher DE, Mohandas N (1996) Kinematics of red cell aspiration by fluorescence-imaged microdeformation. Biophys J 71:1680-1694.

Discher DE, Mohandas N, Evans EA (1994) Molecular maps of red cell deformation: hidden elasticity and in situ connectivity. Science 266:1032-1035.

Drenckhahn D, Schafer T, Prinz M (1985) Actin, myosin, and associated proteins in the vertebrate auditory and vestibular organs: immunocytochemical and biochemical studies. In: Auditory biochemistry (Thomas CC, ed), pp 317-335. Springfield, IL: Dreschers.

Evans EA (1973) New membrane concept applied to the analysis of fluid shear- and micropipette-deformed red blood cells. Biophys J 13:941-954.

Evans EA, Skalak R (1979) Mechanics and thermodynamics of biomembranes: part 1. CRC Crit Rev Bioeng 3:181-330.

Flock A, Flock B, Ulfendahl M (1986) Mechanisms of movement in outer hair cells and a possible structural basis. Arch Otorhinolaryngol 243:83-90.

Fluhler E, Burnham VG, Loew LM (1985) Spectra, membrane binding, and potentiometric responses of new charge shift probes. Biochemistry 24:5749-5755.

Forge A (1991) Structural features of the lateral walls in mammalian cochlear outer hair cells. Cell Tissue Res 265:473-483.

Gale JE, Ashmore JF (1994) Charge displacement induced by rapid stretch in the basolateral membrane of the guinea-pig outer hair cell. Proc R Soc Lond [Biol] 255:243-249.

Gulley RL, Reese TS (1977) Regional specialization of the hair cell plasmalemma in the organ of Corti. Anat Rec 189:109-123.

Hallworth R, Evans BN, Dallos P (1993) The location and mechanism of electromotility in guinea pig outer hair cells. J Neurophysiol 70:549-558.

Holley MC (1996) Outer hair cell motility. In: The cochlea (Dallos P, Popper AN, Fay RR, eds), pp 386-434. New York: Springer.

Holley MC, Ashmore JF (1988a) A cytoskeletal spring in cochlear outer hair cells. Nature 335:635-637.

Holley MC, Ashmore JF (1988b) On the mechanism of a high-frequency force generator in outer hair cells isolated from the guinea pig cochlea. Proc R Soc Lond [Biol] 232:413-429.

Holley MC, Ashmore JF (1990) Spectrin, actin, and the structure of the cortical lattice in mammalian cochlear outer hair cells. J Cell Sci 96:283-291.

Holley MC, Kalinec F, Kachar B (1992) Structure of the cortical cytoskeleton in mammalian outer hair cells. J Cell Sci 102:569-580.

Ikeda K, Takasaka T (1993) Confocal laser-scanning microscopical image of the distribution of intracellular organelles in cochlear outer hair cells. Jpn J Physiol 43[Suppl 1]:S171-S173.
Iwasa KH (1993) Effect of stress on the membrane capacitance of the auditory outer hair cell. Biophys J 65:492-498.

Iwasa KH (1994) A membrane motor model for the fast motility of the outer hair cell. J Acoust Soc Am 96:2216-2224.

Iwasa KH (1996) Membrane motor in the outer hair cell of the mammalian ear. Comments Theor Biol 4:93-114.

Iwasa KH, Chadwick RS (1992) Elasticity and active force generation of cochlear outer hair cells. J Acoust Soc Am 92:3169-3173.

Kakehata S, Santos-Sacchi J (1995) Membrane tension directly shifts voltage dependence of outer hair cell motility and associated gating charge. Biophys J 68:2190-2197.

Kakehata S, Santos-Sacchi J (1996) Effects of salicylate and lanthanides on outer hair cell motility and associated gating charge. J Neurosci 16:4881-4889.

Kalinec F, Holley MC, Iwasa KH, Lim DJ, Kachar B (1992) A membrane-based force generation mechanism in auditory sensory cells. Proc Natl Acad Sci USA 89:8671-8675.

Lipsky NG, Pagano RE (1985) A vital stain for the Golgi apparatus. Science 228:745-747.

Nakagawa T, Kakehata S, Akaike N, Komune S, Takasaka T, Uemura T (1994a) Voltage-dependent channels in dissociated outer hair cells of the guinea pig. Eur Arch Otorhinolaryngol 251:S57-S60.

Nakagawa T, Kakehata S, Yamamoto T, Akaike N, Komune S, Uemura T (1994b) Ionic properties of $I_{\mathrm{k}, \mathrm{n}}$ in outer hair cells of guinea pig cochlea. Brain Res 661:293-297.

Nakagawa T, Oghalai JS, Saggau PS, Brownell WE (1997) Voltagesensitive dye experiments in outer hair cells with patch-clamp. Abstracts of the Twentieth Midwinter Meeting of the Association for Research in Otolaryngology, p 292.

Pollice PA, Brownell WE (1993) Characterization of the outer hair cell's lateral wall membranes. Hear Res 70:187-196.

Raphael Y, Athey BD, Wang Y, Lee MK, Altschuler RA (1994) F-actin, tubulin, and spectrin in the organ of Corti: comparative distribution in different cell types and mammalian species. Hear Res 76:173-187.

Ruggero MA, Rich NC (1991) Furosemide alters organ of Corti mechanics: evidence for feedback of outer hair cells upon the basilar membrane. J Neurosci 11:1057-1067.

Saito K (1983) Fine structure of the sensory epithelium of guinea-pig organ of Corti: subsurface cisternae and lamellar bodies in the outer hair cells. Cell Tissue Res 229:467-481.

Santos-Sacchi J (1991) Reversible inhibition of voltage-dependent outer hair cell motility and capacitance. J Neurosci 11:3096-3110.

Santos-Sacchi J (1993) Harmonics of outer hair cell motility. Biophys J 65:2217-2227.

Santos-Sacchi J, Dilger JP (1988) Whole-cell currents and mechanical responses of isolated outer hair cells. Hear Res 35:143-150.

Shehata W, Brownell WE, Dieler R (1991) Effects of salicylate on shape electromotility and membrane characteristics of isolated outer hair cells from guinea pig cochlea. Acta Otolaryngol 111:707-718.

Sit PS, Lue A, Spector AA, Popel AS, Brownell WE (1997) Micropipette aspiration on the outer hair cell lateral wall. Biophys $\mathbf{J}$ 72:2812-2819.

Slepecky N (1989) Cytoplasmic actin and cochlear outer hair cell motility. Cell Tissue Res 257:69-75.

Spector AA, Brownell WE, Popel AS (1996) A model for cochlear outer hair cell deformations in micropipette aspiration experiments: an analytical solution. Ann Biomed Eng 24:241-249.

Spector AA, Brownell WE, Popel AS (1997a) Elastic properties of the composite outer hair cell wall. Ann Biomed Eng, in press.

Spector AA, Brownell WE, Popel AS (1997b) Mechanical and electromotile characteristics of auditory outer hair cells. J Cell Eng, in press.

Tolomeo JA, Steele CR, Holley MC (1996) Mechanical properties of the lateral cortex of mammalian auditory outer hair cells. Biophys J 71: 421-429. 